\title{
Estrés académico en estudiantes que cursan asignaturas de Física en ingeniería: dos casos diferenciados en Colombia y México
}

\author{
Academic stress in students coursing Physics subjects in Engineering: two differentiated \\ cases in Colombia and Mexico
}

\section{Estresse acadêmico em estudantes que curtam sujeitos de Física em Engenharia: dois casos diferenciados na Colômbia e no México}

\author{
Oscar Jardey Suárez ${ }^{1}$ \\ Mario Humberto Ramírez-Díaz ${ }^{2}$
}

Recibido: marzo 2020

Aceptado: agosto 2020

Para citar este artículo: Suárez, O. J., Ramírez-Díaz, M. H. (2020). Estrés académico en estudiantes que cursan asignaturas de Física en ingeniería: dos casos diferenciados en Colombia y México. Revista Científica, 39(3), 341-352. https://doi.org/10.14483/23448350.15989

\section{Resumen}

Este artículo reporta el estudio de las variables subyacentes al estrés académico en dos poblaciones diferenciadas de estudiantes citadinos que cursan asignaturas de Física en instituciones de educación superior (Colombia y México). El inventario utilizado es el de Polo, Hernández y Pozo (1996), el cual mide dos dimensiones: respuestas al estrés y actividades que producen estrés. Se ha considerado una dimensión adicional que considera aspectos propios de la clase de Física. La población de estudiantes universitarios que participaron en cada comunidad fue de 124 por país. El instrumento tiene una confiabilidad de 0,918 (Col) y 0,937 (Mxn); la medida de adecuación muestral de Kaiser-Meyer-Olkin fue de 0,838 (Col) y 0,878 (Mxn); la varianza interpretada $61,40 \%$ (Col) y $64,46 \%$ (Mxn). Los resultados muestran cuatro variables subyacentes a la población de estudiantes en Colombia (respuestas al estrés [RE], interacción [I], la evaluación [E] y actividad escolar extra [AEE]); en tanto que en México hay tres variables subyacentes al estrés (respuestas al estrés [RE], la evaluación [E] y entorno escolar [EE]). Las respuestas al estrés tienen diferencias entre las poblaciones. La evaluación, al igual que otros estudios, se muestra como una variable que subyace al estrés académico en ambas poblaciones; sin embargo, su comprensión muestra diferentes fuentes.

Palabras clave: enseñanza de la ingeniería, estrés académico, evaluación, Física educativa, rendimiento académico.

\begin{abstract}
This document reports the study of the variables underlying academic stress in two differentiated populations of city students who study physics in the engineering faculty (Colombia and Mexico). The inventory used is that of Polo, Hernández and Pozo of
\end{abstract}

1. Fundación Universidad Autónoma de Colombia. oscar.jardey.suarez@gmail.com

2. Instituto Politécnico Nacional, México, D.F. mramirezd@ipn.mx 
1996 that measures two dimensions: stress responses and stress-producing activities. An additional dimension that considers aspects of the physics class has been considered. The population of university students who participated in each community in each 124 for country. The instrument has a reliability of 0,918 (Col) and 0,937 (Mxn), the Kaiser-Meyer-Olkin sample adequacy measure, 0,838 (Col) and 0,878 (Mxn), the interpreted variance $61,40 \%$ (Col) and $64,46 \%$ (Mxn). The results show four underlying variables to the student population in Colombia (stress responses [RE] Interaction [I] Evaluation [E] and Activity Extra School [AEE]) while in Mexico there are 3 underlying variables stress (Stress Responses [RE], Evaluation [E] and School Environment $[E E])$. The stress responses have differences between populations. The evaluation, like other studies, is shown as a variable that underlies academic stress in both populations, however their understanding shows different sources.

Keywords: academic performance, academic stress, engineering education, evaluation, physics education.

\section{Resumo}

Este documento trata sobre o estudo das variáveis subjacentes ao estresse acadêmico em duas amostras distintas de estudantes nativos que cursam disciplinas de física em Instituições de Ensino Superior (Colômbia e México). O instrumento utilizado é o de Polo, Hernández e Pozo (1996), que mede duas dimensões: respostas ao estresse e atividades que produzem estresse. Considerou-se uma dimensão adicional que trata sobre aspectos próprios da aula de física. A amostra de estudantes universitários que participaram de cada comunidade foi de 124 em cada país. O instrumento tem uma confiabilidade de 0.918 (Col) e 0.937 (Mxn), a medida de adequação amostral de Kaiser-Meyer-Olkin, 0.838 (Col) e 0.878 (Mxn) e a variância interpretada 61.40 \% (Col) e $64.46 \%$ (Mxn). Os resultados mostram 4 variáveis subjacentes à amostra de estudantes na Colômbia (Respostas ao Estresse [RE], Interação [I], a Avaliação [E] e a Atividade Escolar Extra [AEE]), enquanto que no México há 3 variáveis subjacentes ao estresse (Respostas ao Estresse [RE], a Avaliação [E] e Ambiente Esco$\operatorname{lar}[\mathrm{EE}])$. As respostas ao estresse apresentam diferenças entre as amostras. A avaliação, tal como em outros estudos, se apresenta como uma variável que subjaz ao estresse acadêmico em ambas as amostras, no entanto, sua compreensão mostra diferentes origens.

Palavras-chaves: Avaliação, educação em física, ensino de engenharia, estresse acadêmico, rendimento acadêmico

\section{Introducción}

Desde diferentes puntos de vista como la psicología (Martínez-Miguélez, 2009), la Iglesia (Deneulin, 2019) o la educación (Hernández-Jiménez, 2015) el ser humano es considerado multidimensional.

El estrés académico (EA), conocido en ocasiones como ansiedad académica, se ha empezado a considerar como un tema de estudio relevante en escenarios propios de la educación. Trabajos previos han estado enfocados en estudiantes en áreas de la salud; sin embargo, algunos otros se han aproximado a la comprensión del EA en otras áreas como Física o Matemáticas (Suárez et al., 2019).

El EA se identifica como un factor que influye en forma inversa con el rendimiento académico en estudiantes universitarios (Monzón, 2007; Pozos-Radillo et al., 2015) de carreras de la salud (Celis et al., 2001; Martínez-Otero, 2014; Toro-Añel et al., 2011). En este sentido, no se han encontrado investigaciones referidas al EA en estudiantes de ingeniería.

Identificar el EA como un factor predictor del logro de aprendizaje ha conllevado a diversos resultados. De una parte, aquellos que señalan que ocasiona interferencia en las actividades, pero no afecta el rendimiento académico (Caldera-Montes et al., 2007; Domínguez-Castillo et al., 2015), hasta otros en los que señalan los elementos predictores del EA (Caldera-Montes et al., 2017).

La ansiedad académica o EA ha sido estudiado e identificado como factor predictor del rendimiento académico en adolescentes, en los casos en que los padres están separados (Casado-Lozano et al., 2013). De otro lado, la ansiedad negativa 
(distrés) puede incidir inversamente en la asertividad (González et al., 2012).

En el contexto de la ingeniería, Cano et al. (2016) identificaron que en la asignatura de ecuaciones diferenciales se presenta una alta repitencia, lo que motivó a estudiar el EA, encontrando que las evaluaciones y la sobrecarga académica conjugada con el poco tiempo para su desarroIlo desatan el EA. Los síntomas son de tipo psicológico, seguido de los físicos y, por último, los comportamentales.

Gómez et al. (2016) analizan las percepciones de los estudiantes de Ingeniería Mecánica en el Instituto Tecnológico de Pachuca, con el interés de comprender su efecto en el rendimiento académico. El instrumento diseñado en el proceso valora la percepción de los estudiantes en relación con aspectos académicos, salud, economía, acoso (matoneo o sexual), familiares entre otros. La conclusión señala que los estudiantes perciben que a medida que avanzan en su carrera profesional sienten mayor nivel de estrés en la actividad académica. Si bien el rendimiento académico no se afecta significativamente por los niveles presentes de EA, sí es una realidad a tratar en el desarrollo de su formación.

Este trabajo pretende abordar la pregunta ¿qué variables, semejantes y diferentes, subyacen al EA en dos grupos de estudiantes diferenciados, en México y Colombia, que cursan asignaturas de Física en la educación universitaria?

\section{Referentes teóricos}

La noción de estrés aparece con el trabajo del inglés Robert Hooke, quien en el siglo XVII estudió los cuerpos que se deformaban elásticamente al ser sometidos a fuerzas externas. En ese mismo sentido, Tomas Young analizó la relación entre el "esfuerzo de tensión" y la "deformación por tensión", lo que posteriormente se conoció como el módulo de Young. De lo anterior, considerando que la Física tenía fuerte incidencia científica, se avanza en el proceso de adaptación de la noción de estrés a otras áreas del conocimiento (Román-Collazos y Hernández-Rodríguez, 2011).

Selye (1946) identificó algunos síntomas similares en pacientes, independientemente de la situación de salud que padecieran, basado en un ejercicio de observación sistemático propone que el sistema hormonal de defensa se activa en diferentes situaciones que enfrenta el sujeto. Además, el conjunto de reacciones sistemáticas no especificadas son el producto de una exposición prolongada y continua al estrés, a lo que denominó como el síndrome de adaptación general (Selye, 1950).

El síndrome de adaptación general es estructurado en tres momentos: reacción, resistencia y agotamiento. En este los síntomas de daño pasivo inespecífico están estrechamente mezclados con la defensa activa. Biológicamente, el estrés es la interacción entre el daño y la defensa, equiparable en Física a la presión que representa una acción recíproca entre la fuerza y la resistencia ofrecida (Selye, 1950).

Desde la psicología, el estrés es objeto de estudio. Pues se considera como una respuesta fisiológica, endocrina o psicológica del cuerpo ante eventos o situaciones que agobian o se perciben amenazantes, provocando síntomas de agotamiento, disminución de apetito, disminución del peso u otros síntomas inespecíficos (Barraza-Macías, 2006; Domínguez-Castillo et al., 2015; Maturana y Vargas, 2015; Nájera-Ruiz y Fernández-Contreras, 2011).

La escuela, en el sentido más general, es un espacio físico o no físico, sincrónico o asincrónico, en el que se presentan situaciones propias de la actividad e interacción escolar. Aquellas situaciones que desarrollan el estrés en una persona, que potencialmente le puede afectar la realización de las actividades académicas, es lo que puede denominarse EA (Barraza y Silerio, 2007; Barraza-Macías, 2006). Desde un punto de vista sistémico, según Águila (2015), se configura como un proceso adaptativo y psicológico que emerge cuando el estudiante está inmerso en una situación que para él es estresante; no obstante, las situaciones 
estresantes son relativas a cada estudiante. Cuando las personas inmersas en los actos educativos se ven sujetas a un conjunto de demandas que, bajo su propia percepción, superan los recursos para afrontarlas se produce un desequilibrio sistémico, que se denomina estresor o situación estresante académicamente. Esta se manifiesta en uno o varios síntomas o reacciones y es allí cuando se requieren acciones de afrontamiento para regresar al equilibrio sistémico.

En la comprensión del EA debe evitarse que sea asumido solamente como algo negativo que debe evitarse. Una taxonomía del EA señala que este puede ser: normal, distrés y eustrés. Algunas actividades requieren de las personas un estrés, que se denomina "estrés normal"; cuando la actividad causa un desequilibrio sistémico, se le denomina distrés; pero, en ocasiones, la persona necesita estrés para desarrollar a cabalidad las actividades, este tipo de estrés es Ilamado eustrés (Barraza y Silerio, 2007; Berrio-García y Mazo-Zea, 2012; Caldera-Montes et al., 2007; Naranjo-Pereira, 2009).

La ansiedad es una noción muy cercana al estrés. De hecho, hay regiones en las que se entrelazan al punto de dificultar su diferenciación. Es preciso señalar, antes de continuar avanzando en la comprensión de los conceptos, que no se pretende dar una definición de de estrés y ansiedad, mucho menos se busca agotar el debate de los especialistas.

La ansiedad se considera como una reacción del estrés que puede resultar beneficiosa en algunas situaciones; pues se entiende que pone a la persona en estado de alerta necesario para atender algunas situaciones que potencialmente se interpretan como amenazas. Podría decirse que la ansiedad es una respuesta adaptativa. La ansiedad es entendida como una emoción, la cual puede converger con diferentes manifestaciones cognitivas, fisiológicas o motoras (Sierra et al., 2003).

Comprender la ansiedad como una emoción tiene algunas similitudes en cuanto la reacción con otro tipo de emociones como lo son la alegría, la tristeza o el miedo, entre otras, tal como las caracterizan Sierra et al. (2003) o la Sociedad Española para el Estudio de la Ansiedad y el Estrés (Seas).

Ante una situación de estrés las personas pueden presentar diferentes tipos de ansiedad: desde una leve hasta una que afecte totalmente su estilo de vida. Cuando una persona ve afectada su interacción social por temas de ansiedad con, por ejemplo, ataques de pánico frecuentes, su estado de ansiedad puede entenderse como grave a severo y se califica como trastorno de ansiedad (Asociación Americana de Psiquiatría, 2019) 3 $^{3}$ Sin embargo, este trabajo no pretende abordar la ansiedad que desarrollan las personas con trastornos.

La investigación se enmarca en el estudio del estrés derivado de las actividades académicas que se realizan en el desarrollo de los cursos de Física a nivel universitario, al que se denomina EA. Los casos de ansiedad severa o estrés patológico que acarrean trastornos no son objeto de este estudio.

\section{Metodología}

Esta parte de la investigación tiene un enfoque cuantitativo. El objetivo principal es identificar las variables subyacentes al EA en dos poblaciones diferenciadas de estudiantes citadinos, los cuales cursan asignaturas de Física en educación superior (Colombia y México) utilizando el método de análisis de componentes principales (ACP).

\section{Participantes}

Los estudiantes que participaron voluntariamente del estudio se distribuyeron así: de México 124; en tanto que en Colombia 395, de los cuales se seleccionó un número igual al de los participantes en México. El requisito para participar es que fuera estudiante activo y que cursase alguna asignatura de Física en modalidad presencial. Las asignaturas podían ser Física I, Física II, Mecánica de Partículas, Mecánica de Sólidos y Fluidos, Electromagnetismo, Física Moderna, Circuitos, entre otras. El semestre

3. Dirección electrónica https://www.psychiatry.org/patients-families/ anxiety-disorders/expert-q-and-a 
por el que se indagó es el que los estudiantes cursan de acuerdo con el pensum en cada universidad.

Cada institución de educación superior tiene en su desarrollo diferentes cortes para la realización de examen, evaluación o parcial. La medición se hizo en el momento en que se finalizaba un corte.

El equipo investigador decidió hacer que los participantes de cada país, Colombia y México, fuese equiparable; por lo que se hizo para los estudiantes un muestreo aleatorio para seleccionar los 124 participantes. El muestreo se hizo en SPSS 18.0 ®. En la tabla 1 está la distribución de los participantes por país y sexo.

Tabla 1. Distribución de estudiantes

\begin{tabular}{ccc} 
Sexo & Colombia & México \\
Hombre & 85 & 72 \\
& $69 \%$ & $58 \%$ \\
Mujer & 39 & 52 \\
& $31 \%$ & $42 \%$ \\
\hline
\end{tabular}

Fuente: elaboración propia de los autores con el apoyo de SPSS 18.0 ®.

\section{Instrumento}

La investigación se apoya en el inventario de Hernández et al. (1996). Esta prueba ha sido utilizada en múltiples investigaciones, las cuales incorporan variables relacionadas con el estrés (Arribas-Marín, 2013; Berrio-García y Mazo-Zea, 2012; García-Ros et al., 2012; Pozos-Radillo et al., 2014), en poblaciones de educación media o preparatoria (Caldera-Montes et al., 2017), así como de educación universitaria (Luna, 2015).

El inventario indaga 23 afirmaciones (Hernández et al., 1996), pero en este estudio se utilizó una versión ampliada que adiciona cinco afirmaciones propias de la dinámica de las clases de Física (Suárez et al., 2019). En síntesis, el inventario consta de 28 afirmaciones distribuidas en tres categorías a priori: situaciones escolares, respuestas al estrés y elementos epistemológicos-didácticos de la Física. En la tabla 2 están las categorías de las respectivas afirmaciones que componen el instrumento. Cada una de las afirmaciones es autoevaluada por los participantes en una escala de uno a siete. El auto reporte se hace considerando los valores: uno (no me produce estrés) y siete (me produce total estrés), para las categorías situaciones estresantes y elementos epistemológicos-didácticos de la Física; y uno (nunca) y siete (siempre) para las respuestas al estrés.

La recolección de la información se hizo de forma mixta dependiendo de los estudiantes. Algunos diligenciaron el auto reporte en papel y otros a través de la internet en un formulario hecho en Google Docs ${ }^{\circledR}$ que se publicó en la página de una de las universidades y cuyo enlace también se promovió a través de los medios electrónicos disponibles. Cuando los participantes diligenciaron en papel el auto reporte, el equipo investigador digitalizó la entrevista. El auto reporte en línea requería el correo electrónico, el cual luego fue cotejado.

\section{Procedimiento para el análisis de datos}

En el procesamiento de los registros se atendieron los siguientes momentos: consolidación de la base de datos, limpieza de datos (verificación del cumplimiento de las condiciones para los participantes), equiparamiento de la cantidad de participantes por país, verificación del índices de fiabilidad (Cronbach, 1951) y, finalmente, utilización del método de ACP para identificar las variables subyacentes al EA.

\section{Resultados}

El inventario de Hernández et al. (1996) obtiene índice de fiabilidad en la medida arriba de 0,900, como se observa en la tabla 3; lo que permite indicar que es muy adecuado en la medida. Al respecto, es necesario indicar que en el espectro de respuesta se amplió el intervalo, que estaba de uno a cinco y pasó de uno a siete. Adicionalmente, se utilizó la adecuación localizada en la Física y las cinco afirmaciones propias de la actividad de la enseñanza de la Física. 
Tabla 2. Inventario de EA usado en la investigación

\begin{tabular}{|c|c|c|}
\hline \multirow[t]{4}{*}{ Categoría } & N. ${ }^{\circ}$ & Afirmación \\
\hline & A1 & Realización de una evaluación de Física (parcial, examen final, quiz). \\
\hline & A2 & $\begin{array}{l}\text { Exposición de trabajos en clase de Física (temas nuevos, sustentación de talleres, } \\
\text { tareas, informe de actividad de laboratorio, entre otros). }\end{array}$ \\
\hline & A3 & $\begin{array}{l}\text { Participación en clase (responder a una pregunta del profesor, realizar preguntas, } \\
\text { entre otros). }\end{array}$ \\
\hline \multirow{8}{*}{ 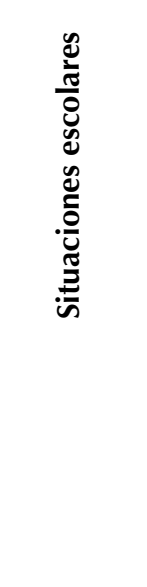 } & A4 & Asistir a tutorías (con profesores o monitores académicos de Física). \\
\hline & A5 & $\begin{array}{l}\text { Sobrecarga académica (excesivo número de créditos, trabajos obligatorios, entre } \\
\text { otros). }\end{array}$ \\
\hline & A6 & Exceso de estudiantes en la clase de Física. \\
\hline & A7 & $\begin{array}{l}\text { Falta de tiempo para poder cumplir con las actividades académicas de la clase de } \\
\text { Física. }\end{array}$ \\
\hline & A8 & Competitividad entre compañeros en la clase de Física. \\
\hline & A9 & $\begin{array}{l}\text { Realización de trabajos obligatorios para aprobar Física (pequeñas investigaciones, } \\
\text { ejercicios en clase, experimentos, construir cosas, redactar el trabajo, entre otros). }\end{array}$ \\
\hline & A10 & $\begin{array}{l}\text { Las tareas de Física (ejercicios, talleres extraclase, informe de actividades de } \\
\text { laboratorio, entre otras). }\end{array}$ \\
\hline & A11 & Trabajar en grupo en la clase de Física. \\
\hline \multirow{8}{*}{ 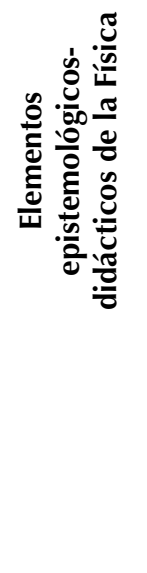 } & A12 & Espacio físico para la clase de Física. \\
\hline & A13 & Convivencia con compañeros de estudio en la clase de Física. \\
\hline & A14 & Dificultades tecnológicas en clase de Física (no tener el recurso o no saberlo usar). \\
\hline & A15 & Expresar por escrito lo que pienso al desarrollar una actividad de Física. \\
\hline & A16 & Comprender las explicaciones al consultar textos de Física. \\
\hline & A17 & Me preocupo en la clase de Física. \\
\hline & A18 & El corazón me late muy rápido o me falta el aire y la respiración es agitada. \\
\hline & A19 & $\begin{array}{l}\text { Realizo movimientos repetitivos con alguna parte de mi cuerpo, me quedo } \\
\text { paralizado o mis movimientos son torpes. }\end{array}$ \\
\hline \multirow{9}{*}{ 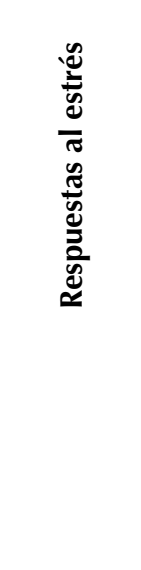 } & A20 & Siento miedo. \\
\hline & A21 & Siento molestias en el estómago. \\
\hline & A22 & Fumo, como o bebo demasiado. \\
\hline & A23 & Tengo pensamientos o sentimientos negativos. \\
\hline & A24 & Me tiemblan las manos o las piernas. \\
\hline & A25 & Me cuesta expresarme verbalmente o a veces tartamudeo. \\
\hline & A26 & Me siento inseguro de mí mismo. \\
\hline & $\mathrm{A} 27$ & Se me seca la boca y tengo dificultades para comer. \\
\hline & A28 & Siento ganas de llorar. \\
\hline
\end{tabular}

Fuente: elaboración propia de los autores con base en el trabajo de Hernández et al. (1996). 
Tabla 3. Índices de fiabilidad del inventario de Hernández et al. (1996) con adecuaciones en la enseñanza de la Física.

\begin{tabular}{|c|c|c|c|}
\hline & Alfa de Cronbach & Colombia & México \\
\hline $\begin{array}{l}\text { Estadísticos } \\
\text { de fiabilidad }\end{array}$ & $\begin{array}{l}\text { Alfa de Cronbach } \\
\text { basada en los } \\
\text { elementos tipificados }\end{array}$ & 0,924 & 0,938 \\
\hline
\end{tabular}

Fuente: elaboración propia de los autores con SPSS $18.0{ }^{\circledR}$.

Al reducir las variables con $\mathrm{ACP}$, usando el método Varimax con Kaiser, se obtuvo que convergió en seis iteraciones con los datos de Colombia, en tanto que en cinco con los datos de México. La tabla 4 contiene los indicadores de los supuestos para cada una de las bases de datos, encontrándose valores equiparables para la medida de adecuación muestral de Kaiser-Meyer-Olkin.

El método ACP aplicado para cada conjunto de datos arrojó que para la población de Colombia se agrupan en cuatro variables subyacentes (respuestas al estrés [RE], interacción [I], la evaluación [E] y actividad escolar extra [AEE]); en tanto que para México en tres variables (respuestas al estrés [RE], la evaluación [E] y entorno escolar [EE]). La tabla 5 tiene los diferentes valores de los resultados. El mapa de cómo se agrupan las afirmaciones en las componentes se puede observar en la tabla 6.

Tabla 4. KMO y prueba de Bartlett

\begin{tabular}{lcll}
\hline $\begin{array}{l}\text { Medida de adecuación muestral de } \\
\text { Kaiser-Meyer-Olkin }\end{array}$ & Colombia & México \\
& 0,838 & 0,878 \\
$\begin{array}{l}\text { Prueba de } \\
\text { Chfericidad de }\end{array}$ & aproximado & 1310,267 & 1185,548 \\
Bartlett & gl & 210 & 136 \\
& Sig. & 0 & 0 \\
\hline
\end{tabular}

Fuente: elaboración propia de los autores con SPSS $18,0 \circledR$.

En las dos poblaciones las variables en las que se coincide son: la respuesta al estrés RE y la evaluación E. La RE es una variable a apriori, en tanto que otros estudios señalan la evaluación como una variable que desata el EA. Sin embargo, como se verá, se avanza en la identificación de las fuentes del EA. A continuación, se describen las variables comunes y posteriormente las otras.

\section{Componente "Respuesta al estrés" (Colombia [C1]-México [C1])}

Las poblaciones expresan su respuesta al estrés en dos aspectos cognitivos y motores. La inseguridad

Tabla 5. Resultados de aplicar la técnica de ACP en los participantes de Colombia y México

\begin{tabular}{|c|c|c|c|c|c|c|c|}
\hline \multirow{2}{*}{ País } & \multirow{2}{*}{ Componente } & \multicolumn{3}{|c|}{$\begin{array}{l}\text { Sumas de las saturaciones al } \\
\text { cuadrado de la extracción }\end{array}$} & \multicolumn{3}{|c|}{$\begin{array}{l}\text { Suma de las saturaciones al } \\
\text { cuadrado de la rotación }\end{array}$} \\
\hline & & Total & $\begin{array}{r}\% \text { de la } \\
\text { varianza }\end{array}$ & $\begin{array}{c}\% \\
\text { acumulado }\end{array}$ & Total & $\begin{array}{r}\% \text { de la } \\
\text { varianza }\end{array}$ & $\begin{array}{c}\% \\
\text { acumulado }\end{array}$ \\
\hline \multirow{4}{*}{ Colombia } & RE & 7,257 & 34,556 & 34,556 & 4,642 & 22,107 & 22,107 \\
\hline & I & 2,924 & 13,924 & 48,48 & 3,285 & 15,643 & 37,750 \\
\hline & E & 1,577 & 7,511 & 55,991 & 2,722 & 12,961 & 50,711 \\
\hline & AEE & 1,136 & 5,409 & 61,400 & 2,245 & 10,689 & 61,400 \\
\hline \multirow{3}{*}{ México } & RE & 7,150 & 42,061 & 42,061 & 5,207 & 30,631 & 30,631 \\
\hline & E & 2,197 & 12,921 & 54,983 & 2,978 & 17,520 & 48,150 \\
\hline & $\mathrm{EE}$ & 1,610 & 9,472 & 64,455 & 2,772 & 16,304 & 64,455 \\
\hline
\end{tabular}

Fuente: elaboración propia de los autores con SPSS 18,0 ${ }^{\circledR}$. 
Tabla 6. Matrices de componentes rotados para México y Colombia con los factores de extracción

\begin{tabular}{|c|c|c|c|c|c|c|c|c|}
\hline \multicolumn{4}{|c|}{ México } & \multicolumn{5}{|c|}{ Colombia } \\
\hline & \multicolumn{3}{|c|}{ Componente } & & \multicolumn{4}{|c|}{ Componente } \\
\hline & $\mathrm{C} 1$ & $\mathrm{C} 2$ & $\mathrm{C} 3$ & & $\mathrm{C} 1$ & $\mathrm{C} 2$ & C3 & $\mathrm{C} 4$ \\
\hline A18 & 0,874 & & & A24 & 0,813 & & & \\
\hline A24 & 0,838 & & & A26 & 0,784 & & & \\
\hline A21 & 0,812 & & & A28 & 0,776 & & & \\
\hline A27 & 0,759 & & & A20 & 0,770 & & & \\
\hline A28 & 0,755 & & & A25 & 0,745 & & & \\
\hline A23 & 0,741 & & & A21 & 0,725 & & & \\
\hline A19 & 0,730 & & & A23 & 0,718 & & & \\
\hline A26 & 0,658 & & & A13 & & 0,825 & & \\
\hline A5 & & 0,774 & & $\mathrm{~A} 12$ & & 0,809 & & \\
\hline $\mathrm{A} 1$ & & 0,719 & & A11 & & 0,764 & & \\
\hline A16 & & 0,698 & & A8 & & 0,623 & & \\
\hline A2 & & 0,641 & & A4 & & 0,601 & & \\
\hline A17 & & 0,613 & & A7 & & & 0,823 & \\
\hline A13 & & & 0,858 & A5 & & & 0,670 & \\
\hline A12 & & & 0,826 & $\mathrm{~A} 1$ & & & 0,645 & \\
\hline A11 & & & 0,725 & A17 & & & 0,514 & \\
\hline \multirow[t]{4}{*}{ A14 } & & & 0,663 & A14 & & & 0,510 & \\
\hline & & & & A9 & & & & 0,824 \\
\hline & & & & A10 & & & & 0,728 \\
\hline & & & & A16 & & & & 0,609 \\
\hline
\end{tabular}

Fuente: elaboración propia de los autores con SPSS $18.0{ }^{\circledR}$.

y los pensamientos negativos, así como las molestias en el estómago o las piernas, acompañadas de un síntoma afectivo como Ilorar. También indican la estrecha multi-reacción que puede evidenciarse en el EA en dos poblaciones como las estudiadas. Sumado a las anteriores reacciones fisiológicas, al parecer también se afectan otras como el miedo y la expresión verbal. Esto señala que al identificarse cualquiera de las anteriores reacciones en algún estudiante potencialmente se puede entender que está sufriendo de EA en esa actividad propia del estudio de la Física. Una vez identificada la situación es importante apoyar con actividades que contribuyan a manejar las situaciones y reacciones al estrés.

\section{Componente "Evaluación" (Colombia [C3]- México [C2])}

Ambas poblaciones se preocupan en la clase de Física durante el momento de la evaluación y quizá una de las fuentes puede obedecer a la sobrecarga académica. Al parecer, en la población de México la dificultad para comprender los temas de Física se da cuando se abordan los textos para estudiar y se hace la presentación de trabajos en la 
clase de Física. Estas situaciones provocan el EA y, por supuesto, son actividades que forman parte de la compresión de la evaluación. En Colombia, la falta de tiempo y las dificultades tecnológicas son dos elementos adicionales.

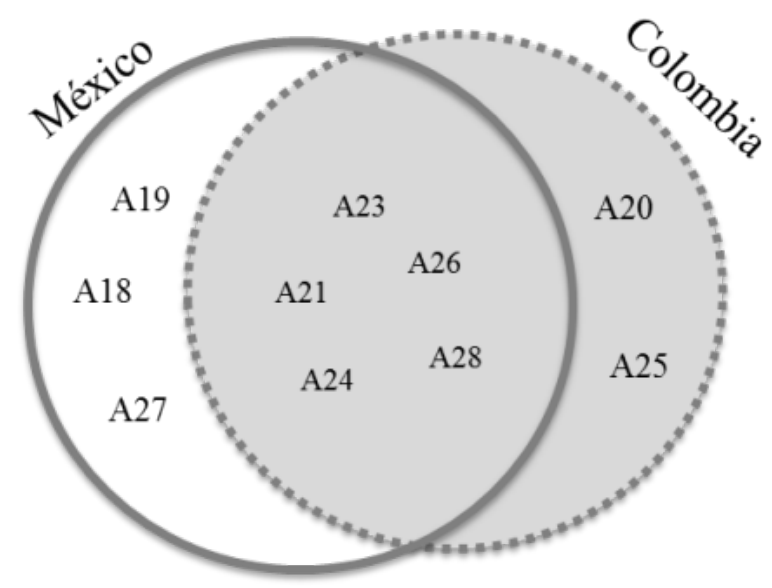

Figura 1. Agrupaciones de respuestas al estrés en Colombia y México.

Fuente: elaboración propia de los autores.

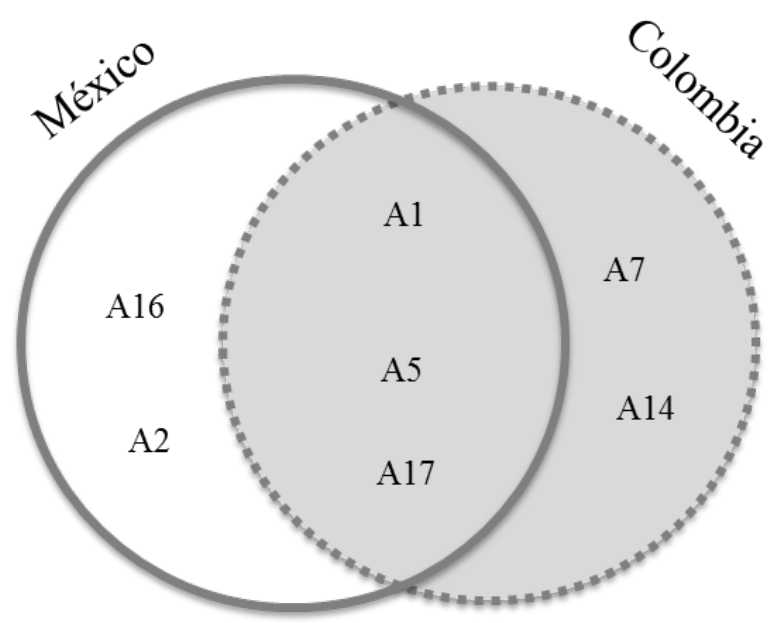

Figura 2. Agrupaciones de evaluación en Colombia y México.

Fuente: elaboración propia de los autores.

Una vez identificadas las fuentes que desarrollan el EA, corresponde revisar críticamente el acto educativo para aportar estrategias que mitiguen el distrés. Es decir, parte de las herramientas para lidiar con esto quizás esté en la formación de hábitos de estudio, ocupación del tiempo libre, autorregulación, entre otras actividades que permitan organizar las actividades del curso de Física y prever lo necesario para llegar a la evaluación. Así mismo, incluir en la actividad docente formas de leer libros de Física o la búsqueda de recursos educativos alternos que suplan o complementen las jornadas de estudio para la preparación de las actividades orientadas a la exposición de ejercicios o a la evaluación.

Si bien preocuparse no está mal, dado que puede hacer parte del "estrés normal" (eustrés) necesario para abordar las actividades del curso de Física, también es preciso potenciar estrategias que permitan convertir esta situación mediante el afrontamiento cuando esta forma parte de un distrés.

\section{Componente "Entorno Escolar" (México [C3])}

La convivencia, el espacio físico, el trabajo en grupo y las dificultades tecnológicas confluyen. Luego, es deseable que el trabajo en grupo se transforme en equipos de trabajo. Esto quiere decir que, en coincidencia con muchos de los perfiles profesionales declarados en la educación superior, apostar a un conjunto de estudiantes con una sana convivencia, con espacios físicos adecuados y apostando por el uso apropiado de la tecnología puede contribuir en un espacio de formación positivo.

En lo referido a la actividad docente, desde el punto de vista didáctico y epistemológico, considerar con claridad las actividades de la clase de Física para trabajo en equipo no es un trabajo menor.

\section{Componente "Interacción" (Colombia [C2])}

Para los participantes de Colombia el trabajo en grupo, el espacio físico y la convivencia se enlazan con la competitividad y la asistencia a tutorías. Luego, las actividades en clase o extraclase para trabajo en grupo, o equipo, resulta interesante y como una estrategia no menor a considerar. La estrategia de trabajo en grupo va de la mano con la competencia propia de la formación en la 
educación superior y se debe prever un acompañamiento orientado a clarificar las inquietudes que en el desarrollo de la actividad emerjan. Dicho lo anterior, la estrategia promueve la interacción entre los estudiantes-saber-profesores.

\section{Componente "Actividad escolar extra" (Colombia [C4])}

Los trabajos obligatorios o tareas de Física provocan que los estudiantes acudan a los textos de Física; asimismo, el hecho de que serán revisados y calificados por el profesor es una variable que es fuente de EA. En este caso, no se pretende omitir las actividades necesarias y propias de las clases de Física, pero se puede contribuir a los estudiantes en proveer las herramientas de afrontamiento y ayudas necesarias que permitan que este estrés sea el normal o necesario para el desarrollo óptimo de las tareas, el aprendizaje y, en consecuencia, la aprobación del curso. El acudir a las explicaciones de los textos de Física aumenta el EA, luego considerar recursos educativos que tengan un formato distinto al texto en papel puede resultar prudente; así, por ejemplo, un conjunto de vídeos, tutorías sincrónicas cara a cara o mediadas por la tecnología, pueden resultar adecuadas en las estrategias ofrecidas por las áreas que prestan servicios educativos.

Las variables latentes en las personas, en México y Colombia, que participaron aportan información relevante en la comprensión del EA que producen las actividades que son calificables y que permitirán aprobar el curso. Estas variables permiten pensar en un conjunto de estrategias con respecto al estudiante, profesor e institución que aporten a los estudiantes las herramientas para afrontamiento de las fuentes de EA que desequilibren negativamente la capacidad de las personas que cursan asignaturas de Física.

\section{Conclusiones}

Se ha encontrado en varios trabajos que el EA tiene una relación inversa con el rendimiento académico o la obtención de logro de aprendizaje. Este trabajo aporta elementos empíricos y reflexiones que contribuyen en la comprensión del EA, que lo lleve de ser un obstáculo para convertirse en un conjunto de oportunidades formativas y de estrategias docentes en la enseñanza de la Física.

En el acto educativo la evaluación es un elemento que se hace a través de diferentes momentos, que en forma diferenciada son fuentes de EA y que potencialmente puede afectar de manera negativa el logro de aprendizaje y, finalmente, la nota que el estudiante obtenga en el curso de Física. En este sentido, el presente trabajo permite señalar la necesidad de ver, en al menos desde tres actores, la situación: el estudiante, el profesor y la institución.

Resulta importante para el estudiante identificar las respuestas a las situaciones que generan el EA, así como establecer el momento en que los recursos que él dispone para afrontarlas son superados por las demandas de las situaciones. Esto para que pueda pedir ayuda y optar por actividades tendientes a mejorar esa situación.

Lo anterior puede conllevar a que, institucionalmente, se propongan un conjunto de estrategias y actividades que aporten a los estudiantes herramientas de autorregulación, organización y planeación de actividades, así como el aprovechamiento del tiempo libre, oferta de acompañamiento extraclase y demás que contribuyan en preparar anticipadamente las situaciones que desarrollan negativamente el EA.

Pensar las actividades que conduzcan al aprendizaje del estudiante resulta una actividad no menor para el profesor (como responsable de la actividad docente). En ellas, aprovechar la actividad en equipo, ya sea tanto en actividades de laboratorio como teóricas, propicia escenarios en los que confluyen aspectos de orden contextual e interactivo que bajo condiciones de EA normal o favorable son necesarias para tener en consideración. La flexibilidad razonable del profesor en la exigencia de cumplimiento es un elemento necesario en el desarrollo de las actividades propuestas 
en los cursos de Física; lo anterior entendiendo la sobrecarga académica o los tiempos limitados, que son resultado de la acumulación de actividades.

\section{Agradecimiento}

El equipo agradece al Sistema Unificado de Investigaciones SUI de la Universidad Autónoma de Colombia y al Centro de Investigación en Ciencia Aplicada y Tecnología Avanzada CICATA del Instituto Politécnico Nacional el apoyo recibido para adelantar trabajos de investigación en colaboración.

\section{Referencias}

Arribas-Marín, J. (2013). Hacia un modelo causal de las dimensiones del estrés académico en estudiantes de Enfermería. Revista de Educacion, 360, 533-556. https://doi. org/10.4438/1988-592X-RE-2011-360-126

Barraza, A., Silerio, J. (2007). El estrés académico en alumnos de educación media superior: un estudio comparativo. Investigación Educativa, 7, 48-65.

Barraza-Macías, A. (2006). Un modelo conceptual para el estudio del estrés académico. Revista Electrónica de Psicología Iztacala, 9(3), 110-129.

Berrio García, N., Mazo Zea, R. (2012). Estrés académico. Revista de Psicología, 3(2), 81-87. https://doi.org/10.1007/s13398-014-0173-7.2

Caldera-Montes, J., Pulido-Castro, B., Martínez-Gonzáles, M. (2007). Niveles de estrés y rendimiento academico en estudiantes de la carrera de Psicología del centro Universitario de los Altos. Revista de Educacion y DesarroIIo, 7, 77-82.

Caldera-Montes, J., Reynoso-González, O., Gómez-Covarrubia, N., Mora-García, O., Anaya-González, B. (2017). Modelo explicativo y predictivo de respuestas de estrés académico en bachilleres. Ansiedad y el Estrés, 23, 20-26. https://doi.org/10.1016/j.anyes.2017.02.002
Cano-lbarra, S., Medina-Torres, M., Ramos-Beltrán, J. (2016). Análisis del estrés académico en estudiantes de ingenier+ia como estrategia para el aprendizaje significativo. Revista Electrónica ANFEI Digital, 2(5), 1-8.

Casado-Lozano, I., Hernández-Sande, A., Manzano-Bazaga, E. (2013). Autoconcepto: factores e influencias. ReiDoCrea: Revista Electrónica de Investigación y Docencia Creativa, 2(2), 138-142.

Celis, J., Bustamante, M., Cabrera, D., Cabrera, M., Alarcón, W., Monge, E. (2001). Ansiedad y estrés académico en estudiantes de medicina humana del primer y sexto año. Anales de La Facultad de Medicina, 62, 25-30. https://doi. org/10.15381/anales.v62i1.4143

Cronbach, L. J. (1951). Coefficient alpha and the internal structure of tests. Psychometrika, 16(3), 297-334. https://doi.org/10.1007/BF02310555

Deneulin, S. (2019). El desarrollo humano integral: una aproximación desde la tradición social católica y el enfoque de las capacidades de Amartya Sen. Revista de Estudios Sociales, 67, 74-86. https://doi.org/https://doi. org/10.7440/res67.2019.06

Domínguez-Castillo, A., Guerrero-Walker, G., Domínguez-Castillo, J. (2015). Influencia del estrés en el rendimiento academico de un grupo de estudiantes universtarios. Educacion y Ciencia, 4, 31-40.

García-Ros, R., Pérez-González, F., Pérez-Blasco, J., Natividad, L. A. (2012). Evaluación del estrés académico en estudiantes de nueva incorporación a la universidad. Revista Latinoamericana de Psicología, 44(2), 143-154.

Gómez, A., Mejía, M., Luna, L. (2016). Efectos del estrés en el desempeño académico de los estudiantes de Ingeniería Mecánica del Tecnológico de Pachuca, Revista de Sistemas y Gestión Educativa, 3(9), 27-36.

González, M. D. L. L., Leal, D., Segovia, C., Arancibia, V. (2012). Autoconcepto y Talento: una relación que favorece el logro académico. 
Psykhe, 21(1), 37-53. https://doi.org/10.4067/ $\underline{\text { S0718-22282012000100003 }}$

Hernández-Jiménez, D. (2015). Educación : una visión desde las dimensiones del ser humano y la vida. Acta Académica, 57, 79-92. http://www.corteidh.or.cr/tablas/r34530.pdf

Hernández, J. M., Polo, A., Pozo, C. (1996). Inventario de estrés académico. Servicio de Psicología Aplicada U.A.M.

Luna, R. (2015). Adaptación del inventario de estrés académico en población universitaria mexicana. Psicología y Salud, 21, 3137 https://doi.org/10.13140/RG.2.1.3146.7123

Martínez-Miguélez, M. (2009). Dimensiones básicas de un desarrollo humano integral. Polis, Revista de la Universidad Bolivariana, 8(23), 119-138. https://doi.org/10.4067/ S0718-65682009000200006

Martínez-Otero, V. (2014). Ansiedad en estudiantes universitarios : estudio de una muestra de alumnos de la facultad de educación. Ensayos Revista de la Facultad de Educación de Albacete, 2(29), 63-78.

Maturana, A., Vargas, A. (2015). El estrés escolar. Revista de Medicina Clinica Condes, 26(1), 34-41. https://doi.org/10.1016/j. rmclc.2015.02.003

Monzón, I. M. M. (2007). Estrés académico en estudiantes universitarios. Apuntes de Psicología, 25(1), 87-99.

Nájera-Ruíz, F., Fernández-Contreras, T. (2011). El estrés académico y sus tensiones en la escuela normal; equilibrio entre demanda y resistencia. En, Estrés, Burnout y bienestra subjetivo Investigaciones sobre salud mental de los agentes educativos. Instituto Universitario Anglo Español ReDIE.

Naranjo-Pereira, M. (2009). Una revision teórica sobre el estres y algunos aspectos relevantes de éste en el ambito educativo. Revista Educacion, 33(2), 171-190. https://doi.org/10.15517/revedu.v33i2.511

Pozos-Radillo, B. E., Preciado-Serrano, M. D. L., Acosta-Fernández, M., Aguilera-Velasco, M. D. L. Á., Delgado-García, D. D. (2014). Academic stress as a predictor of chronic stress in university students. Psicologia Educativa, 20(1), 47-52. https://doi.org/10.1016/j.pse.2014.05.006

Pozos-Radillo, B. E., Preciado-Serrano, M. D. L., Rosa, A., Campos, P., Acosta-Fernández, M., de los Ángeles Aguilera, V. M. (2015). Estrés académico y síntomas físicos, psicológicos y comportamentales en estudiantes Mexicanos de una universidad pública. Ansiedad y Estrés, 21(1), 35-42.

Román-Collazos, C., Hernández-Rodríguez, Y. (2011). El estrés académico: una revisión crítica del concepto desde las ciencias de la educación. Revista Electrónica de Psicología Iztacala, 14(2), 1-14.

Selye, H. (1946). The General Adaptation Syndrome and the Disease of adaptation. Journal of Clinical Endocrinology, 6, 117. https://doi. org/10.1210/jcem-6-2-117

Selye, H. (1950). Stress And The General Adaptation Syndrome. Brithis Medical Journal, 4666-4678. https://doi.org/10.1136/bmj.1.4667.1383

Suárez, O., Hurtado-Marquez, A., Pulido-Cardozo, O. (2019). Estrés académico en estudiantes de Ingeniería que cursan Física : análisis exploratorio. Revista Científica, número esp, 7683. https://revistas.udistrital.edu.co/index.php/ revcie/article/view/14478

Toro-Añel, A., Gorguet-Pi, C., Pérez-Infante, Y., Ramos-Gorguet, D. (2011). Estrés académico en estudiantes de Medicina de primer año con bajo rendimiento escolar. Medisan, 15(1), 17-22. 